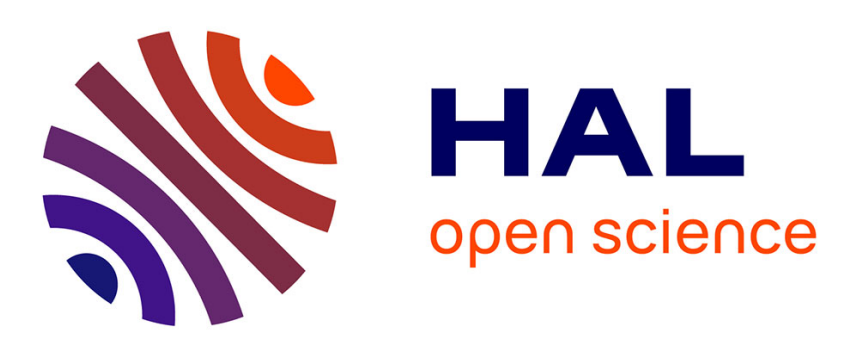

\title{
Micro-to-Nano Biomechanical Modeling for Assisted Biological Cell Injection
}

\author{
Hamid Ladjal, Jean-Luc Hanus, Antoine Ferreira
}

\section{To cite this version:}

Hamid Ladjal, Jean-Luc Hanus, Antoine Ferreira. Micro-to-Nano Biomechanical Modeling for Assisted Biological Cell Injection. IEEE Transactions on Biomedical Engineering, 2013, Vol.99 (N.99), pp.1-11. 10.1109/TBME.2013.2258155 . hal-00821621

\section{HAL Id: hal-00821621 \\ https://hal.science/hal-00821621}

Submitted on 22 May 2013

HAL is a multi-disciplinary open access archive for the deposit and dissemination of scientific research documents, whether they are published or not. The documents may come from teaching and research institutions in France or abroad, or from public or private research centers.
L'archive ouverte pluridisciplinaire HAL, est destinée au dépôt et à la diffusion de documents scientifiques de niveau recherche, publiés ou non, émanant des établissements d'enseignement et de recherche français ou étrangers, des laboratoires publics ou privés. 


\title{
Micro-to-Nano Biomechanical Modeling for Assisted Biological Cell Injection
}

\author{
Hamid Ladjal, Jean-Luc Hanus, Antoine Ferreira (memberIEEE)
}

\begin{abstract}
To facilitate training of biological cell injection operations, we are developing an interactive virtual environment to simulate needle insertion into biological cells. This paper presents methodologies for dynamic modeling, visual/haptic display and model validation of cell injection. We first investigate the challenging issues in the modeling of the biomechanical properties of living cells. We propose two dynamic models to simulate cell deformation and puncture. The first approach is based on the assumptions that the mechanical response of living cells is mainly determined by the cytoskeleton and that the cytoskeleton is organized as a tensegrity structure including microfilaments, microtubules and intermediate filaments. Equivalent microtubules struts are represented with a linear mass-tensor finite element model and equivalent microfilaments and intermediate filaments with viscoelastic Kelvin-Voigt elements. The second modeling method assumes the overall cell as an homogeneous hyperelastic model (St-Venant-Kirchhoff). Both graphic and haptic rendering are provided in real-time to the operator through a 3D virtual environment. Simulated responses are compared to experimental data to show the effectiveness of the proposed physically-based model.
\end{abstract}

Index Terms-Biomechanics - Finite Element Modeling - Cell injection - Haptic interaction

\section{INTRODUCTION}

Cell manipulation is a prevalent process in the field of molecular biology. This process plays an important role in intracytoplasmic sperm injection (ICSI), pronuclei deoxyribonucleic acid (DNA) injection, therapeutic and regenerative medicine, and other biomedical areas. ICSI has progressively replaced all other micro-injection procedures for overcoming intractable male-factor infertility and has emerged in a relatively short time as a routine procedure for many in-vitro procedures. Nevertheless, injections are currently performed manually and technicians are required to be skillful enough to not destruct the cell structure of the ovum during the ICSI process. As a consequence, the success rate remains relatively low and strongly dependant on the experience and skills of the operator [1]. Conventional methods of ICSI injection require the operator to undergo long training (over a period of one year), and the success rates are low (around $10 \%-15 \%)$ due to poor reproducibility. The fragile nature

This paper was presented in part at the IEEE International Conference on Robotics and Intelligent Systems, San Francisco (USA), 2011. This work was supported by the Centre de Recherche en Biologie de Baugy (France).

H. Ladjal is with LIRIS laboratory UMR 5205 F-69622, and IPNL laboratory CNRS UMR 5822 F-69622 Université Claude Bernard Lyon 1, France. Email:hamid.ladjaleliris.cnrs.fr

J-L. Hanus and A. Ferreira are with Laboratoire PRISME, Ecole National Suprieure d'Ingnieurs de Bourges, France jean-luc.hanus, antoine.ferreiradensi-bourges.fr of a biological cell requires the operator to be efficient; otherwise the patrician may damage the cell. There are also risks of contamination due to direct human involvement. The drawbacks involved in conventional methods have motivated the research community to develop robotic-based tools to perform cell injection tasks.

Various cell injection systems have been developed to provide more controllable manipulation of biological cells [2], [3], [4]. If visual servoing is a necessary condition for accurate micro-injection operations, the knowledge of interactive forces acting during pipette insertion plays an important role too since it can be used to provide force feedback for precise regulation of needle penetration speed and strength [5]. Reducing cell deformation and needle deviation will also lower the risk of cell damage. However, when manipulating deformable biological objects, force sensor measurement will provide only the local forces at the pipette puncture point which limits strongly the operator haptic rendering [5]. In order to learn, train and analyze basic cell injection procedures, most operators are aiming to use artificial cells because practicing on human oocyte has ethical concerns and potential risks [6]. The development of computer-based systems using visual and force-feedback for both injection training and injection assistance will help to overcome some of these problems. Similar research on virtual reality environment platforms has been carried out at a macroscopic scale in surgery simulation and modeling of soft tissues [7], [8], [9]. By analogy to in vivo intracytoplasmic sperm injection, similar boundary conditions occur. An important difference is that, within the framework of micro-injection, the needle can be considered as a rigid surgical tool. The cell geometry and biomechanical subcomponents properties (biomembrane, cytoskeleton, cytoplasm, nucleus) are of major importance in simulation and modeling because these factors affect the amount of cell membrane deformation, needle deviation and interaction forces [10], [11].

As a consequence, a physically based nonlinear biomechanical cell model is required to render in a realistic way the interactions between the needle and the cell [11], [12]. The objective of this paper is to develop and implement a biomechanical finite element approach within a virtual environment dedicated to real-time cell injection to facilitate training of ICSI operations. These models include the topological information of the living cells (shape and dimensions), and the biological structure (cytoplasm layers, cytoskeletons and nucleus) [6], [13], [14]. 
We used an explicit nonlinear finite element formulation. First, we propose a dynamic model, based on an equivalent cytoskeleton nanostructure. The developed approach is based on two main assumptions: (i) the mechanical response of living cells is mainly determined by the cytoskeleton and (ii) the cytoskeleton is organized as a tensegrity nanostructure including microfilaments, microtubules and intermediate filaments. Second, we consider the cell as a homogeneous and incompressible solid with viscoelastic properties. The equivalent isotropic cell structure is described by a Saint Venant-Kirchhoff hyperelastic material model. Both models are implemented, tested in a real-time visual and haptic simulator and compared to cell injection experiments. As far we know, no prior complete micro-injection simulation environment that handles both physically-based simulation and real-time visual and haptic feedback has been proposed in the literature.

This paper is organized as follows: in Section II, we present an overview of the mechanical models for living cells. In Section III, we present the biomechanical finite element approach dedicated to real-time injection. In Section IV, we present the methodology of the virtual environment system for cell injection. In Section $V$, we make a comparative study between our real-time cell injection simulator and experimental data. Finally, we give some concluding remarks and the directions for future work.

\section{RELATED RESEARCH}

The development of biological cell models able to simulate insertion forces such as the force peak, latency in the force changes, and separation of different forces such as stiffness and damping force is a challenging issue. The majority of the proposed models are mainly derived into three classes: microscale continuum, energetic and nanoscale structural approaches.

The models belonging to the first class assume the biological cell to be equivalent to a one or two phases continuum model without any insights on molecular nanoscale mechanical properties [15], [16]. The main advantages are their facility to compute the mechanical properties of cells and provides details on the distribution of stresses and strains induced on cells (zebrafish and medaka embryos) at different developmental stages [17],[18]. However, the continuum approach, which views the cell has a tensed balloon filled with molasses or jello [21], has its drawbacks since it is not capable of accounting for the molecular deformations and interactions within the cell.

The second category of models takes into account the contributions of various cytoskeleton structures to the overall energy budget of cell during contraction [19], [20]. It is based on the percolation theory and polymer physics models at large deformations. The advantage of this model is its independence in the choice of its coordinate system and the particular details of the cytoskeleton architecture, because the energy is a scalar quantity. However, its difficult to find an optimal physical correspondence to experimental data.

The third class of models includes the tensegrity structures divided into two subclasses: spectrin-network model and cytoskeletal models for adherent cells. The former deals a specific microstructural network for spectrin cells at large deformations [21]. The later considers the cytoskeleton as the main structural component and attributes a central role to cytoskeleton contractile forces. Recently, a specific architectural model of the cytoskeletal framework (tensegrity) deserved wide attention. The tensegrity approach has described many aspects of cell deformability including nonlinear features of cellular structural behavior. These models view the cell as a network of microfilament, microtubule and actin, that distributes forces within the cell through a balance of compression and tension [21], [25], [26]. These models can imitate a number of features observed in living cells during mechanical tests including prestress-induced stiffening, strain hardening, and the effect of cell spreading on cell deformability [27], [23], [24]. A full mechanocell model consisting of the cell membrane, the nuclear enveloppe and actin filaments described as a combination of various spring elements has also been developped based on the minimum of the elastic energy during deformation [22]. Other studies estimate the viscoelastic properties of the detached and retracting cytoskeleton using time-sequential imaging combined with atomic force microscopy in order to understand cellular dynamics, especially cell migration [28]. The present analysis points out different biomechanical models as candidates for the modeling of an oocyte cell composed of multi-layer material components such as the pellucida zona, the biomembrane and the cytoplasm cytoskeleton (Fig.1). In this study, the micro/nanostructural behavior of the oocyte cell is modeled through two modeling approaches: (i) the nano-scale model (tensegrity model coupled to a viscoelastic membrane) and (ii) the micro-scale model (equivalent homogeneous cell hyperelastic model).
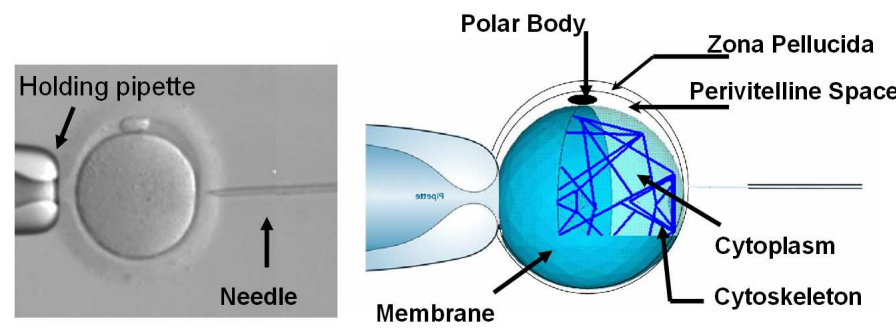

Fig. 1. (a) Biological oocyte cell for micro-injection procedure and (b) associated multilayered biomechanical model.

\section{MicRo-TO-NANO SCALE BiomechanicAL MODELING FOR OOCYTES}

This section presents different micro-to-nano scale biomechanical models to simulate the mechanical response of a biological cell during punction. 


\section{A. Nanometer Scale: Equivalent cytoskeleton structure}

The first model represents the nanostructural behavior of an oocyte cell composed of multilayer material components with a tensegrity model coupled to a viscoelastic membrane. It assumes that (i) the mechanical response of living cells is mainly determined by the cytoskeleton and (ii) the cytoskeleton is organized as a tensegrity nanostructure. As far the authors know, the tensegrity model has never been applied to microrobotic intracellular injection simulation for real-time haptic training purposes.

1) Mechanical and Geometrical Properties: The overall structure of the tensegrity model is described as follows. The cytoskeleton is composed of an interconnected structure of various cross-linked interlinked filamentous biopolymers that extends from the center to the cell surface (bio-membrane). Three major filamentous components compose the cytoskeleton, i.e. the actin microfilaments, microtubules and intermediate filaments which are physically interlinked. The actin stress, plays an important role in many cellular functions, including morphological stability, adhesion, and motility. Because of their central role in force transmission, it is important to characterize the mechanical properties of stress fibers [29]. However, in our studies we focus on properties of whole cells or of actin microfilaments, microtubules and filaments intermediate isolated outside cells [32]. Recent study investigates the initial mechanical response of axonal microtubule bundles under uniaxial tension using a discrete bead-spring representation. Unfortunately, the elements in the system, both microtubule and tau cross-link, were assumed to follow a linear elastic constitutive relationship. This assumption is less valid in an entropic stretching regime, in compressive loading, and potentially in a high force regime [33].

In our simulation, we choose a simplified tensegrity structure (due to real-time simulation constraints) with six compressing struts (two in each orthogonal direction), these struts aggregate the behavior of the microtubules, viewed as beams as shown in Fig.2. These struts are attached to 36 pre-stressed cable segments :

- 12 cables representing the intermediate filaments which are connected to the parallel struts.

- 24 cables representing the actin microfilaments connected to the end points of each strut.

2) Formulation of the microtubule finite element model: In this study, we propose a hybrid model well adapted to the deformable microstructural geometry of the oocyte cell. The biomechanical model we used is a simplified cytoskeleton tensegrity structure where struts are modeled with 3D first order tetrahedral finite elements and cables with viscoelastic Kelvin-Voigt elements. Firstly, we introduce briefly the relation between stresses and strains in the context of hyperelastic materials for large and small deformations, then we present the mass-tensor finite element approach with tetrahedral elements. This non-classical finite element approach is well-suited to perform real-time simulation.

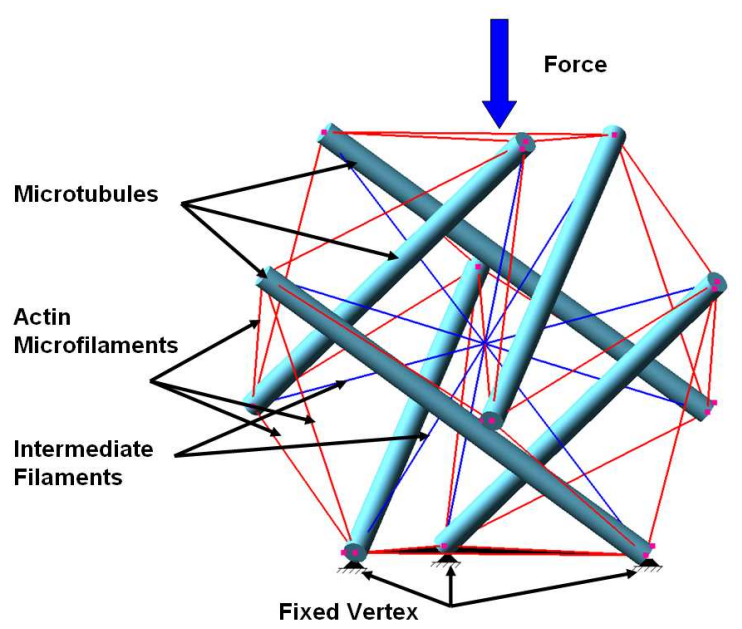

Fig. 2. Three-dimensional finite element tensegrity model of the oocyte cell (membrane surface nodes not represented).

For an isotropic elastic or St Venant-Kirchhoff hyperelastic material the elastic energy, noted $W$, can be written as:

$$
W(\mathbf{E})=\frac{\lambda}{2}(\operatorname{tr} \mathbf{E})^{2}+\mu \operatorname{tr}\left(\mathbf{E}^{2}\right)
$$

where $\mathbf{E}$ is the Green-Lagrange strain tensor $\lambda$ and $\mu$ are the Lame's coefficients

$$
\begin{aligned}
\mathbf{E} & =\frac{1}{2}\left(\mathbf{F}^{T} \cdot \mathbf{F}-\mathbf{I}\right) \\
& =\frac{1}{2}\left(\operatorname{grad} \underline{U}+\operatorname{grad}^{T} \underline{U}+\operatorname{grad}^{T} \underline{U} \cdot \operatorname{grad} \underline{U}\right)
\end{aligned}
$$

A displacement based finite element solution is obtained with the use of the principle of virtual works. Using finite element method (FEM) notations, inside each tetrahedron $T^{\tau}$, the displacement field is defined by a linear interpolation $\left[\mathbf{N}^{\tau}\right]$ of the nodal displacement vector $\left\{\mathbf{u}^{\tau}\right\}$ of the four vertices of tetrahedron:

$$
\left\{\mathbf{U}(\mathbf{x})^{\tau}\right\}=\left[\mathbf{N}^{\tau}(\mathbf{x})\right] \quad\left\{\mathbf{u}^{\tau}\right\}
$$

For small deformations, the Green-Lagrange strain tensor is linearized into the infinitesimal strain tensor:

$$
\epsilon=\frac{1}{2}\left(\operatorname{grad} \underline{U}+\operatorname{grad}^{T} \underline{U}\right)
$$

The relation between the Cauchy stress tensor and the linearized strain tensor is written with Lame's coefficient in condensed vector notation as :

$$
\{\boldsymbol{\sigma}\}=\lambda\left(\{\boldsymbol{\varepsilon}\}_{1}+\{\boldsymbol{\varepsilon}\}_{2}+\{\boldsymbol{\varepsilon}\}_{3}\right)[\mathbf{I}]+2 \mu\{\boldsymbol{\varepsilon}\}
$$

where $[\mathbf{I}]$ is the identity matrix.

The principle of virtual work applied to a single tetrahedron $T^{\tau}$ leads to the elementary stiffness matrix $\left[\mathbf{K}^{\tau}\right]$ such 
that the elementary nodal force vector acting on a tetrahedron is:

$$
\left\{\mathbf{f}^{\tau}\right\}=\left[\mathbf{K}^{\tau}\right]\left\{\mathbf{u}^{\tau}\right\}
$$

This stiffness matrix is composed of a plurality of elementary submatrices each connecting the elementary force acting on the node, $i$, to the displacement of the node, $j$ :

$$
\begin{aligned}
{\left[\mathbf{K}_{\mathbf{i j}}^{\tau}\right]=} & \frac{1}{36 V^{\tau}}\left(\lambda\left\{\mathbf{m}_{\mathbf{i}}\right\}\left\{\mathbf{m}_{\mathbf{j}}\right\}^{T}+\right. \\
& \left.\mu\left\{\mathbf{m}_{\mathbf{j}}\right\}\left\{\mathbf{m}_{\mathbf{i}}\right\}^{T}+\mu\left\{\mathbf{m}_{\mathbf{i}}\right\}^{T}\left\{\mathbf{m}_{\mathbf{j}}\right\}[\mathbf{I}]\right)
\end{aligned}
$$

where $\{\mathbf{m}\}$ are unit outward-pointing normals to triangular faces and $V^{\tau}$ is the volume of the tetrahedron $T^{\tau}$.

Taking into account the contribution of all adjacent tetrahedra, the global internal force acting on a node $l$ can be expressed as follows:

$$
\left\{\mathbf{F}_{\mathbf{i n t}}^{\mathbf{l}}\right\}^{\text {Struts }}=\sum_{\tau \in \mathcal{V}_{l}}\left(\sum_{j=1}^{4}\left[\mathbf{K}_{\mathbf{i j}}^{\tau}\right]\left\{\mathbf{u}_{\mathbf{j}}\right\}\right)
$$

where $\mathcal{V}_{l}$ is the neighborhood of vertex $l$ (i.e. the tetrahedra containing node $l$ ).

The tensors $\left[\mathbf{K}_{\mathbf{i j}}^{\tau}\right]$, depending on the rest geometry and Lame's coefficients, are constant. They can be pre-computed in an off-line phase. It is the essential advantage of the mass-tensor approach which makes it useful for real-time application.

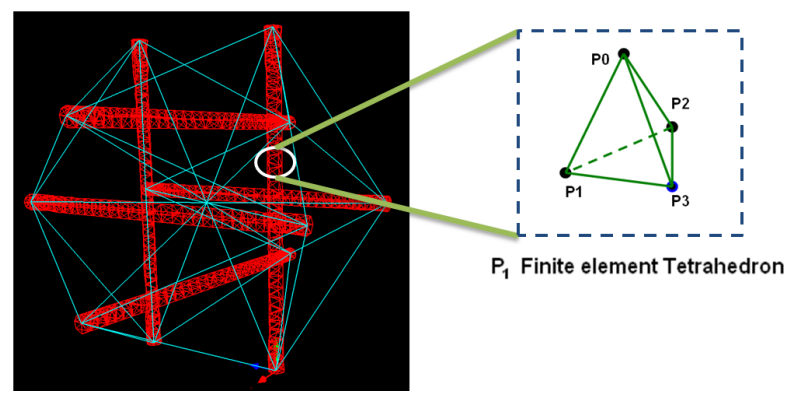

Fig. 3. Using the $P_{1}$ finite element tetrahedron for the struts of the tensegrity model.

3) Cable behavior for the tensegrity structure: In this section, we present the description of the cable behavior about our tensegrity model (Fig.2). These pre-stressed cables are assumed to behave as viscoelastic mass-spring-dampers. Each cable is modeled with two masses interconnected via spring and damper in parallel (Kelvin Voigt model). In the local frame, the relation between the stress and the strain can be written as follows:

$$
\sigma=E \varepsilon+\eta \dot{\varepsilon}
$$

with $\sigma=\frac{F}{S}$ and $\epsilon=\frac{l-l^{0}}{l^{0}}$, where $F$ is the applied load in the extremity of the cable, $l$ and $l^{0}$ and $S$ are respectively the resting length of the cable, the initial length and the section of the cable. We replace these parameters in (9), and we obtain :

$$
\underline{F}_{\text {local }}^{\text {able }}=\left(\frac{E S\left(l-l^{0}\right)}{l^{0}}+\frac{\eta \dot{i}}{l^{0}}\right) \underline{x}
$$

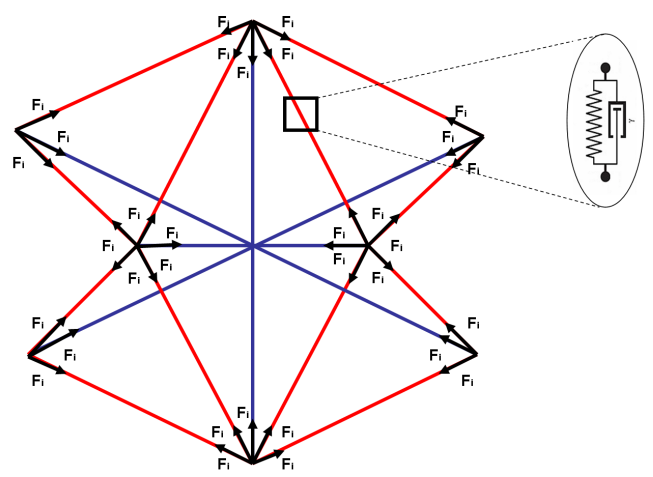

Fig. 4. Mass-spring-damper model for cable structure.

In the global coordinates, for the tensegrity structure, we can expand this equation easily and obtain the force applied in each node by summing the forces contributed by all nodes connected (Fig.4). The force applied to an extremity of the strut of the tensegrity structure is the sum of the forces exerted by the struts and the cables.

\section{B. Micrometer Scale : Equivalent homogeneous cell}

The second model assumes that an oocyte cell could be modeled as a whole by a homogeneous solid, isotropic (visco)hyperelastic and nearly incompressible material properties.

1) Mechanical and Geometrical Properties: The oocyte cell is meshed with 3D first order tetrahedral elements Fig.5. The main geometrical characteristics of the cell were obtained from experimental data. The mesh that has been used for the non real-time simulations, was obtained by a sequence of successive refinements until mechanical convergence. It is constituted by a non regular tetrahedron volume mesh composed of 1115 vertices and 322 tetrahedra where all vertices are free. Only the surface nodes in contact with the holding micropipette are fixed (see Fig.5).

2) Formulation of finite element model: Incompressible and nearly-incompressible materials such as oocyte cell, are difficult to model accurately with a pure displacementbased FE procedure. The simplest hyperelastic (HE) material model is the Saint Venant-Kirchhoff model which is just an extension of the linear elastic material model to the nonlinear regime. In our work, the cells are modeled by the volume object discretized into a conformal tetrahedral mesh as defined by finite element theory. Inside every tetrahedron $T_{k}$, the displacement field is defined by a linear interpolation of the displacement vectors of the four vertices of the tetrahedron 


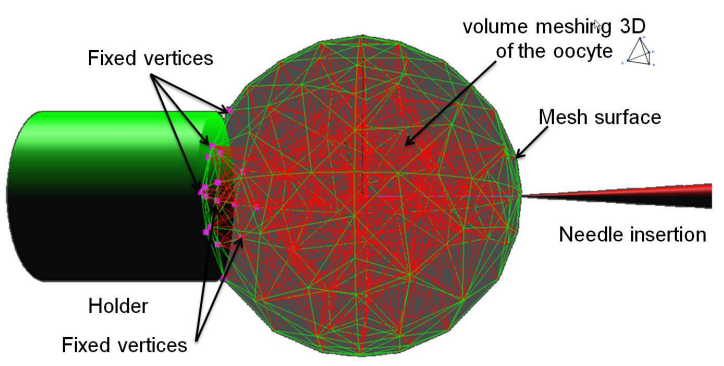

Fig. 5. Three-dimensional finite element model of the mouse oocyte cell.

$T_{k}$ as defined by (1)-(3). Since the strain is quadratic in displacement, the elastic energy is a fourth-order polynomial in displacement. The relation between nodal forces and nodal displacement is nonlinear and can be written as:

$$
\begin{aligned}
& \left\{\mathbf{F}_{\mathbf{i}}^{\tau}\right\}=\underbrace{\sum_{j=1}^{4}\left[\mathbf{K}_{\mathbf{i j}}^{\tau}\right]\left\{\mathbf{u}_{\mathbf{j}}\right\}}_{F_{1}^{\tau}} \\
& +\underbrace{\sum_{j, k=1}^{4}\left\{\mathbf{u}_{\mathbf{k}}\right\}\left\{\mathbf{u}_{\mathbf{j}}\right\}^{T}\left\{\mathbf{C}_{\mathbf{j k i}}^{\tau}\right\}+\frac{1}{2}\left\{\mathbf{u}_{\mathbf{j}}\right\}^{T}\left\{\mathbf{u}_{\mathbf{k}}\right\}\left\{\mathbf{C}_{\mathbf{i j k}}^{\tau}\right\}}_{F_{2}^{\tau}} \\
& +\underbrace{2 \sum_{j, k, l=0}^{3} D_{j k l i}\left\{\mathbf{u}_{\mathbf{l}}\right\}\left\{\mathbf{u}_{\mathbf{k}}\right\}^{T}\left\{\mathbf{u}_{\mathbf{j}}\right\}}_{F_{3}^{\tau}}
\end{aligned}
$$

where the global force can be written as:

$$
\left\{\mathbf{F}_{1}^{\text {int }}\right\}=\sum_{\tau \in \mathcal{V}_{l}}(\underbrace{\left\{\mathbf{F}_{1}^{\mathbf{T}}\right\}}_{\text {Linearforce }}+\underbrace{\left\{\mathbf{F}_{\mathbf{2}}^{\mathbf{T}}\right\}+\left\{\mathbf{F}_{\mathbf{3}}^{\mathbf{T}}\right\}}_{\text {non-linearforce }})
$$

where $\left\{\mathbf{C}_{\mathrm{ijk}}^{\tau}\right\}, D_{i j k l}^{\tau}$ are respectively a vector and a scalar. As these tensors depend only on the rest geometry and Lame's coefficients, they can be pre-computed for real-time application.

\section{Dynamic Model}

The deformation of the deformable object is given by the displacement of the nodes according to the acting external and internal forces. In an interactive simulation the applied forces change in time and the virtual objects have to react to them in real time. Therefore, the FEM solution has to be simulated dynamically. The equation of motion of a vertex $l$ of the cell mesh can be written:

$$
M^{l}\left\{\ddot{\mathbf{u}}_{l}\right\}+\gamma^{l}\left\{\dot{\mathbf{u}}_{l}\right\}+\sum_{\tau \in \mathcal{V}_{l}}\left(\left\{\mathbf{F}_{1}^{\text {int }}\right\}\right)=\left\{\mathbf{F}_{\text {ext }}^{\mathbf{l}}\right\}
$$

where $M^{l}$ and $\gamma^{l}$ are respectively the mass and damping coefficients of each vertex. To solve the dynamic system, we tested different integration schemes (implicit and explicit) taking into account the tradeoff between real-time simulation

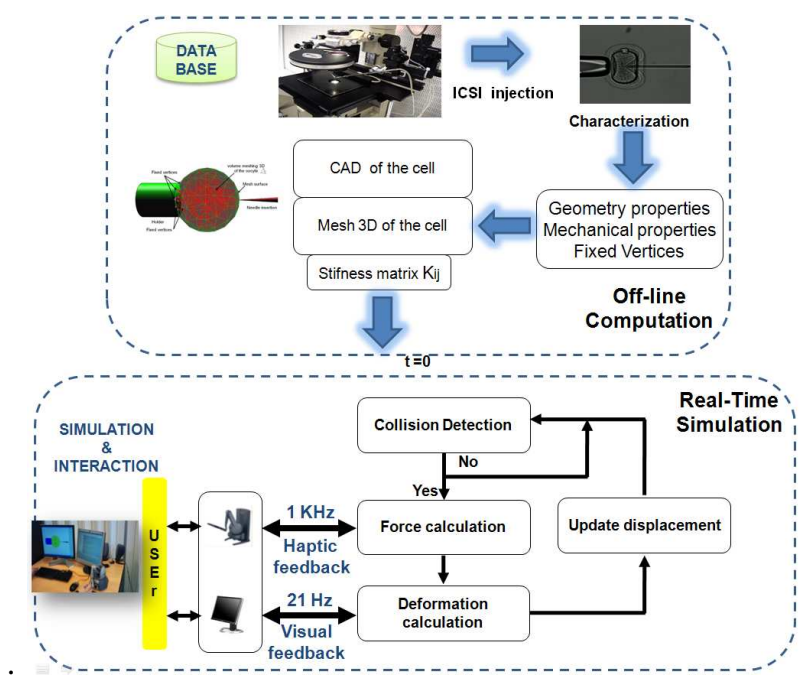

Fig. 6. Computational architecture for simulating force-reflecting deformable cell micro-injection in a virtual environment. The figure shows the two simulation phases used for the real-time micro-injection of the cell: (i) off-line pre-calculations of stiffness matrices and (ii) simulation of visual and haptic interaction.

and haptic stability requirements. We choose the explicit centered finite-difference scheme.

\section{3D REAL-TIME VIRTUAL REALITY BASED ICSI SIMULATOR}

Fig.6 shows an overview of the proposed ICSI simulator. The system includes the computer generated mesh of oocyte, the needle, a collision detection algorithm, the physicallybased model of the deformable cell and the haptic interaction controller. A significant difficulty in using the finite element technique for real-time simulation is the computational cost. These mesh-based methods require time consuming numerical integration operations to perform the system stiffness matrices. As a consequence, to spare computational time, we adopted a computational architecture with two simulation stages: (i) off-line mesh generation and computation of elementary stiffness matrices, (ii) real-time visual and haptic interaction during ICSI simulation.

\section{A. Off-line computation}

The most costly and time-consuming operations are realized during a pre-calculation step. The database contains geometric properties of oocyte cells using the microinjection setup image analysis. The geometry design of oocyte cell models are based on a commercial computeraided design (CAD) package (MARC-ADAMS). The meshing of the 3D internal structure is then carried out through a dedicated 3D meshing software (GID software) modeled in exact dimensions. Although they were displayed as 3D texture-mapped objects to the user, they were modeled as connected line segments to reduce the number of collision computations during real-time interactions. The oocyte cell was approximated as an assembly of discrete 
tetrahedral elements. In order to avoid inverted or very distorted elements due to mesh local large deformation, the initial oocyte mesh is generated with tetrahedral elements pre-stretched in the direction opposite to the expected needle movement. The tetrahedral elements are interconnected to each other through a fixed number of nodes (see Fig.3 and Fig.5). The mechanical (Young modulus,

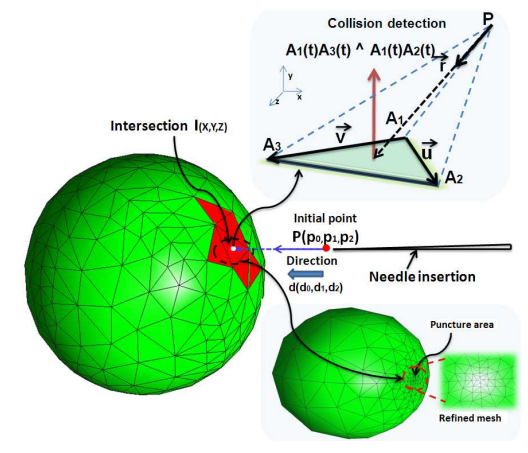

Fig. 7. Scheme illustrating the collision detection algorithm using a simple triangle points intersection problem: needle intersection with triangle surface of the cell, and the refining of the mesh.

Poisson coefficient) and geometrical (diameter, volume) properties of the cell structure and tensegrity parameters are determined by real tests and injected in the FEM to compute all the elementary tensors $\left[\mathbf{K}_{\mathrm{ij}}^{\tau}\right],\left\{\mathbf{C}_{\mathbf{i j k}}^{\tau}\right\}$ and $D_{i j k l}^{\tau}$.

\section{B. Real-time haptics-enable simulator}

We have implemented separate threads to update the loops. During the injection task, the contact between the tip and the cell must occur at a special set of points (nodal points). Collision detection module specifies the type of tool-tissue interaction. The needle, in a virtual environment, can be modeled as a complex 3D object, composed of numerous surfaces, edges and vertices. However, in our implementation, since the needle can be considered as rigid, we used a pointbased representation of the pipette injector. The collision detection is the first step to carry out realistic interactions. However, locating the contact primitive (e.g. facets) between two objects may be computationally expensive, especially if the objects are composed of a large number of polygons. In this case, we employed a simple ray-triangle intersection with local search technique [34]. To optimize the collision detection algorithm and cell deformation, we refine our mesh only in a puncture area. The size of this refined area has been empirically determined as five times the needle diameter. The number of elements in this puncture area is fixed by the mesh density. The scaling factor between the mesh density in the puncture area and the rest of the cell is also fixed at five.

The visual feedback is updated after each displacement calculation step and the haptic feedback is returned after each force calculation step.

\section{RESULTS AND VALIDATION}

\section{A. Experimental results}

In order to test the accuracy and reliability of the proposed user interface system with haptics enabled simulation, we used experimental real data on a mouse oocyte ZP [30]. During experiments, the applied force is measured by a MEMSbased two-axis cellular force sensor [31] which is capable of resolving normal forces applied to a surface as well as tangential forces. The geometrical dimensions are determined by $2 \mathrm{D}$ image processing and reported in Table I. The oocyte is observed to be spherical at rest: a maximum relative out of roundness of $3.7 \%$ and a mean value of $0.5 \%$ were measured. The mechanical properties of the homogenous cell model are determined through a micro-injection setup [30] and reported in Table I. The force indentation curve is shown in Fig.8. The force increases nonlinearly as deformation increases. When the indentation depth of the cell reaches about $45 \mu \mathrm{m}$, the $\mathrm{ZP}$ and the plasma membrane are punctured, the puncturing forces are approximately $7.5 \mu N$. The experimental data are plotted with $95 \%$ prediction bounds (error bounds $5 \%$ ) for more precision (Fig.8). As the mechanical and geometrical properties of the tensegrity model cannot be experimentally evaluated, these properties are settled in Table II using the literature values [32], [35], [36]. These settling parameters are then injected in the finite element simulation.

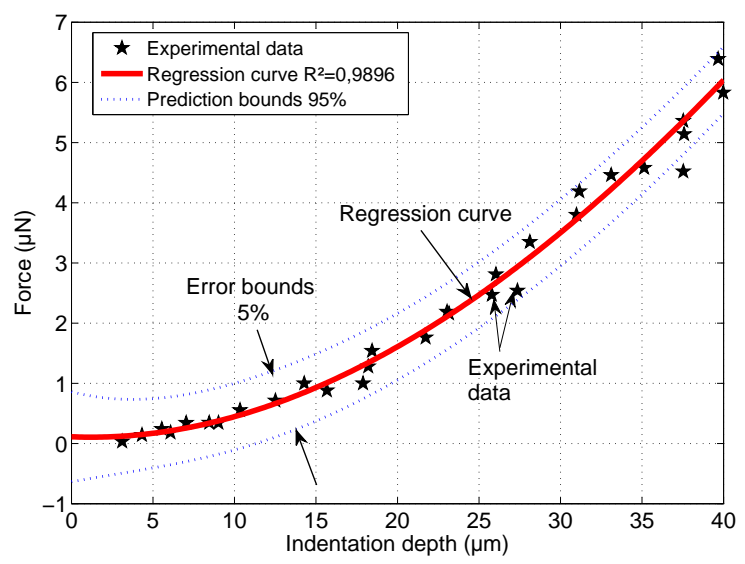

Fig. 8. Experimental data: force versus needle insertion depth of the mouse oocyte cell with $95 \%$ prediction bounds.

\section{B. Validation of haptics-based simulator}

There are two criteria for assessing the suitability of the FE based simulator for the real-time simulation of cell puncture: model accuracy and real-time performance. We validated the model accuracy of our simulator for both FE models (homogeneous hyperelastic model and tensegrity model) against the results from our least-square fitting tests as reported in previous section. To get a trustworthy platform, the relative error between smoothed experimental results and simulated ones should not exceed $5 \%$. 


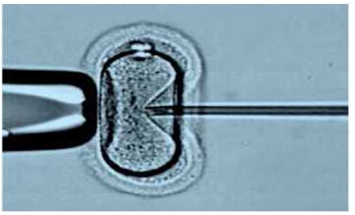

$40 \mu m$ needle insertion

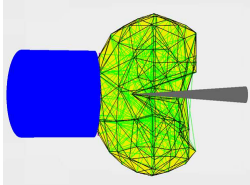

$40 \mu m$ RTS

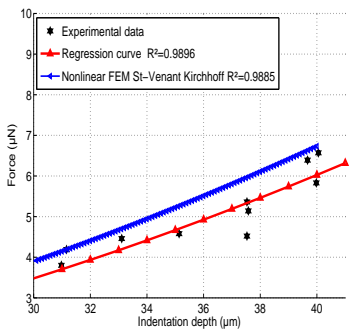

$40 \mu m$ FvD

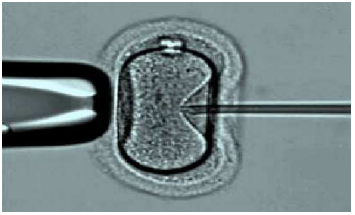

$30 \mu m$ needle insertion

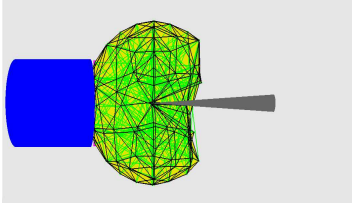

$30 \mu m$ RTS

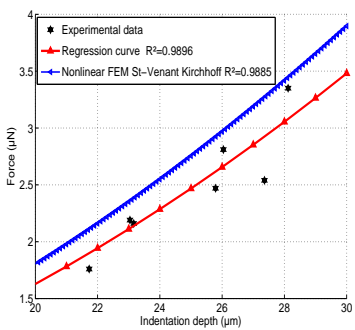

$30 \mu m$ FvD

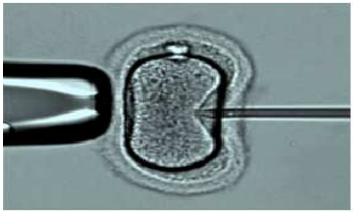

$20 \mu m$ needle insertion

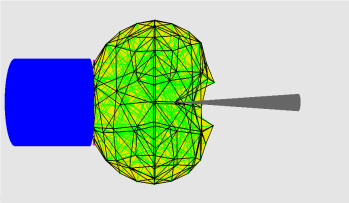

$20 \mu m$ RTS

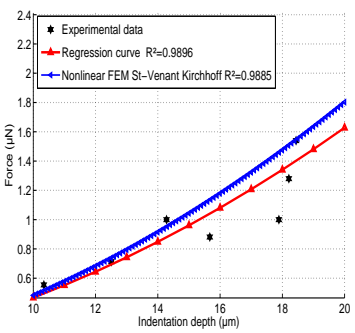

$20 \mu m$ FvD

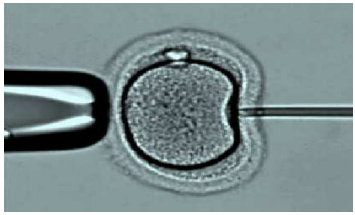

$10 \mu m$ needle insertion

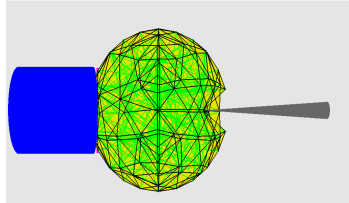

$10 \mu m$ RTS

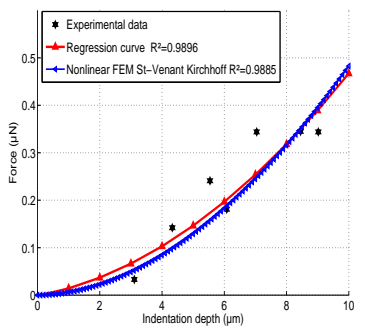

$10 \mu m$ FvD

Fig. 9. Quantitative and qualitative comparison of the various stages of cell deformation of needle insertion between : experimental data, Real-Time Simulations (RTS) and Force versus Displacement (FvD).

TABLE I

MECHANICAL AND GEOMETRICAL PROPERTIES ASSIGNED TO THE HOMOGENEOUS CELL MODEL.

\begin{tabular}{c|c}
\hline & Mechanical and geometrical properties \\
\hline \hline Young modulus & $17.9 \mathrm{KPa}$ \\
Poisson coefficient & 0.49 \\
Diameter & $56 \mu \mathrm{m}$ \\
Mass of the cell & $9.195210^{-5} \mathrm{mg}$ \\
Mesh of the cell & 123 vertices and 379 tetra \\
Cell volume & $91952 \mu \mathrm{m}^{3}$ \\
Maximum indentation depth & $40 \sim 44 \mu \mathrm{m}$ \\
\hline
\end{tabular}

1) Case 1: Equivalent homogeneous cell model: A mesh containing 379 tetrahedral elements and 123 vertices was used for validation purposes. In comparison with the initial refined mesh used in non real-time simulation, the number of elements is decreased so as to afford real-time simulations while ensuring a maximum relative error of $5 \%$ in comparison with the converged refined mesh. The mechanical and geometrical properties used in the finite element simulations are settled in Table.I. The Fig. 10 and Fig. 9 show comparisons between experimental data, linear and non-linear finite element responses. In order to represent a nearly incompressible material behavior, a numerically acceptable value of $\nu=0.49$ was adopted. The reaction force is plotted versus the needle insertion depth. The force feedback simulations shown in Fig. 9 demonstrate that the non-linear FE St-Venant-Kirchhoff model is in good agreement with experimental data. For comparison purposes, it can be clearly seen that a linear finite element model is valid only for small displacements (less than $10 \%$ of the mesh size). The visual realism of the simulation tests depicted in (Fig. 9) demonstrates the good agreement of visual deformation rendered to the operator at various stages of needle insertion.

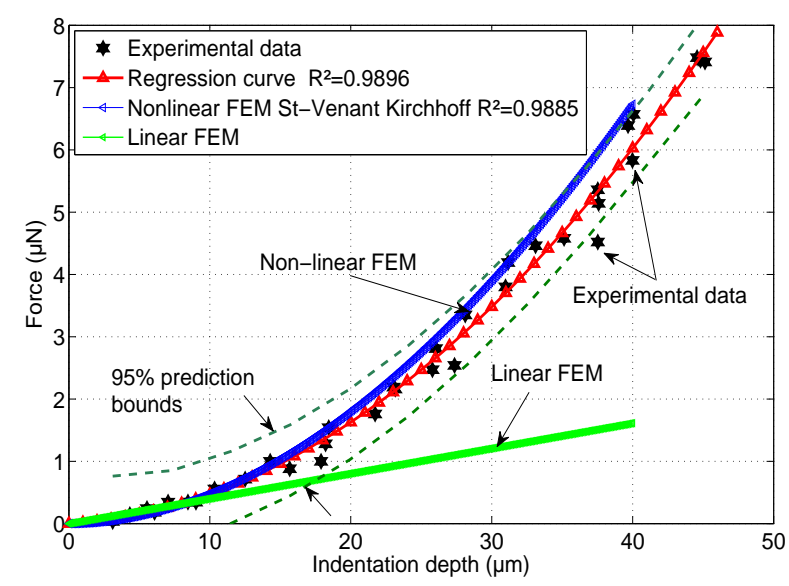

Fig. 10. Force versus indentation depth: comparison between experimental data, non-linear St Venant Kirchhoff and linear finite element simulations.

2) Case 2: Equivalent cytoskeleton structure model: The mesh that was used for the following simulations is shown in Fig. 3. It is constituted by a non regular tetrahedron volume mesh composed of 1014 vertices and 1798 tetrahedrons 
where all vertices are free and only three nodes are fixed (see Fig. 2). It should be noticed that the viscoelastic biomembrane is not represented for simplicity of representation.

TABLE II

MATERIAL AND GEOMETRICAL PROPERTIES ASSIGNED TO THE TENSEgRity MODEL: ACtin Filaments (AF), Micro Tubules (MT) AND INTERMEDIATE FILAMENTS (IF), WHERE BS: BENDING STIFFNESS, S: SECTION OF THE FILAMENTOUS [32].

\begin{tabular}{c||c|c|c}
\hline & AF & MT & IF \\
\hline \hline $\mathrm{E}(\mathrm{Pa})$ & $1.3 \sim 2.6 \times 10^{9}$ & $1.2 \times 10^{9}$ & $0.3 \sim 0.4 \times 10^{9}$ \\
$\nu$ & 0.3 & 0.3 & 0.3 \\
$\mathrm{D}(\mathrm{nm})$ & - & 5 & - \\
$\mathrm{BS}\left(\mathrm{Nm}^{2}\right)$ & $7 \times 10^{-26}$ & $2.6 \times 10^{-23}$ & $4 \sim 12 \times 10^{-27}$ \\
Mesh & cable & 1014 vertices & cable \\
& & 1798 tetrahedra & \\
\hline
\end{tabular}

In figure 11, we show two static qualitative examples of the deformation of a tensegrity structure. Firstly, the penetration axis of the micropipette is supposed to be aligned with the tensegrity geometrical center. The resulting force is applied to the center of one strut of the tensegrity model. Secondly, the penetration axis of the micropipette is supposed to be parallel to the first load but the resulting force is applied in the extremity of a strut. The deformed meshes show the effect of the deformation of one member on the whole tensegrity structure.
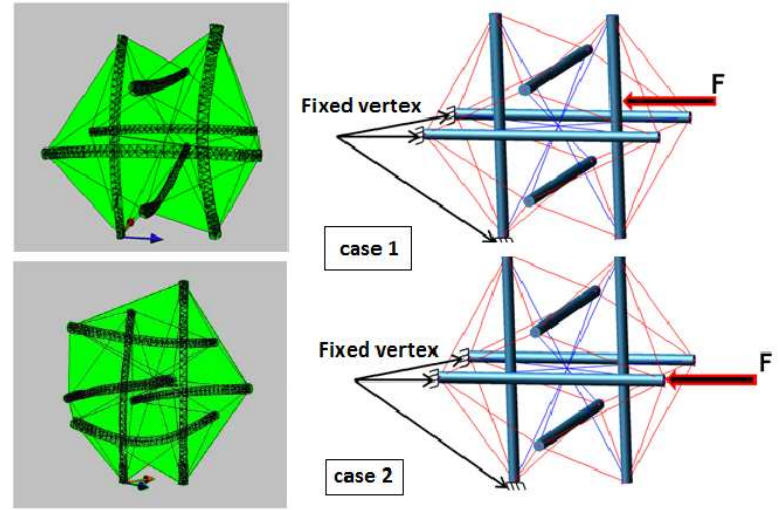

Fig. 11. Deformed tensegrity meshes. Case 1; the force is applied to the middle of a strut, Case 2; the force is applied in the extremity of a strut.

The figure 12 shows the non-linear response of the tensegrity cell model during a needle insertion. The non-linear increase in cell stiffness as a result of increasing prestress exhibited by the model is attributed to the internal cytoskeleton. This effect is also seen when forces are applied at a distance from the underlying cytoskeleton, although the effect is less pronounced. As illustration, the Fig. 13 shows the nonlinear response of the cell tensegrity structure compared to experimental data. These results are qualitatively in good agreement with the experimental results but presents lower deformation forces due to a lack of accuracy in the determination of the Young modulus parameters (microtubules, microfilaments and intermediate filaments).

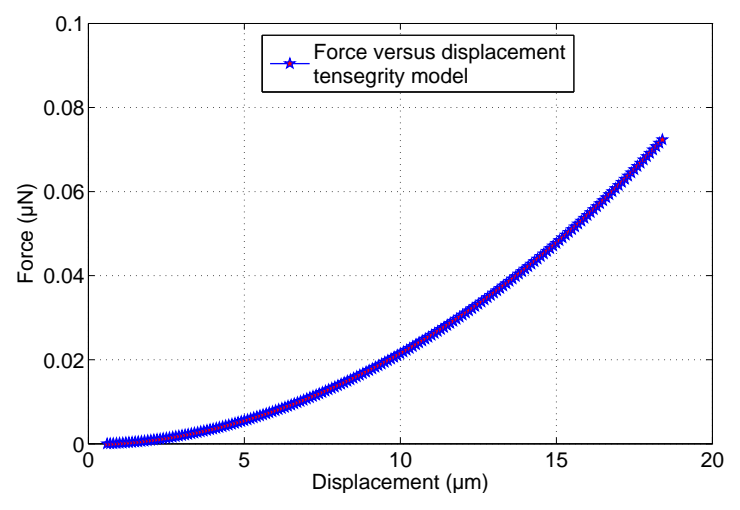

Fig. 12. Force versus needle insertion depth for tensegrity model.

For real-time performance, we need only around 24-25 $\mathrm{Hz}$ screen refresh rate for visual updates, which corresponds to the critical fusion frequency of human eye. However, the human tactile sensory system is much more sensitive, hence the haptic device requires an update rate between $300 \mathrm{~Hz}$ and $1 \mathrm{kHz}$. To test the real-time performance of the standard CPU implementation, we used meshes of different complexity with the number of elements ranging from 100 to 5000. An experimental system was implemented on a personal computer with a Intel Core Duo 3.2 GHz CPU with 4 GB memory, and GeForce 8600 GTS with 512Mo memory. In this experiment, we investigated the time required for pre-computation (offline computation) and the real-time deformation process by changing the number of vertices of a cell model (Table.III). The relationship between the number of vertices and the offline computation time was identified by a nonlinear law while the relation between the number of vertices and the real-time simulation remains quasi-linear (see Fig.14). However, the accuracy of the finite element analysis depends on the mesh refinement. The problem should be solved by using graphics processing unit (GPU) to perform finer mesh simulations.

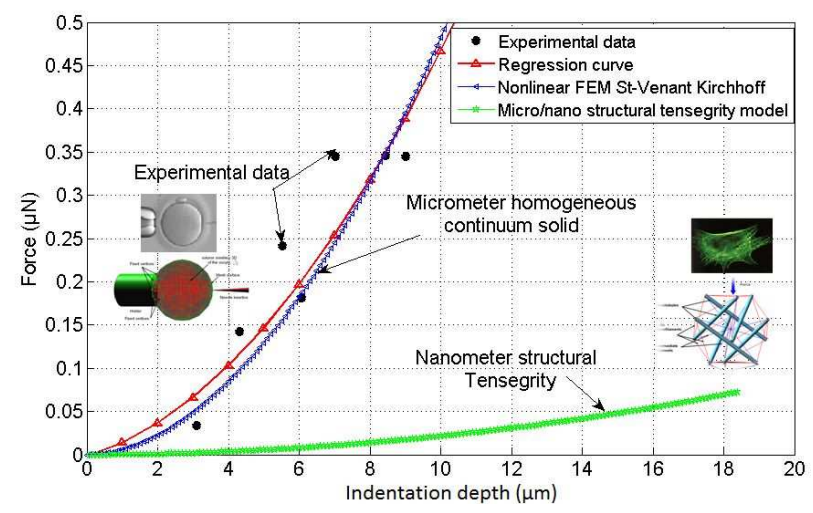

Fig. 13. Force versus indentation depth: comparison between experimental data, micro/nano structural tensegrity model and homogeneous non-linear viscoelastic FE . 


\section{Discussion}

In this paper we have tested different micro-to-nano scale biomechanical modeling methods for computing deformations during simulated micropipette insertion into 3D elastic cells. To assess the performance, cost and accuracy of the biomechanical models, the 3D finite element methods were implemented in a prototype and subjected to computational experiments. The performance of the computational methods is mainly related to the biomechanical cell structure, the material and geometrical properties assigned to the cell models and the coarseness of the mesh.

In this study, we see that a good biomechanical cell model is crucial for making accurate predictions of cell deformations. The experimental force measurements realized by microelectromechanical (MEMS) force sensors [37],[38] rendered possible the identification of global cell mechanical properties with a microNewton resolution. It fully validates the proposed equivalent homogeneous cell hyperelastic model at micro-scale (validity of the linear model restricted to cell deformations less than 5\%). Compared to the microinjection model based on membrane theory proposed by [17], we obtain similar force-deformation relationship demonstrating the effect of the injector radius, the membrane stress and tension distributions, changes of the internal pressure, and the deformed cell shape. However, the measurement of material and geometrical properties of the multilayer material components composing the oocyte structure necessitates in situ mechanical characterization tools with nanoNewton force resolutions. As shown in Fig.13, the computational results obtained by applying forces to the model tensegrity nodes suggest a key role for the cytoskeleton in determining cellular stiffness [35]. As experimentally confirmed in [38], the role of the cytoskeleton in stiffening a cell during the process of cell maturation (i.e. young or old oocytes) can be clearly demonstrated. The proposed equivalent tensegrity model captures nonlinear structural behaviors such as strain hardening and prestress effects, and variable compliance along the cell surface but only reflects $10 \%$ of the measured punction force. The lack of such stiffening in our model may reflect the simplistic nature of how we have estimated the Young's modulus of tensegrity components (microtubule, microfilaments and intermediate filaments). The material and geometrical properties presented in Table II are related to eukaryotic adherent cells properties due to the lack of experimental measures in cell oocytes. The Fig.13 shows that it do not fully reflects the biomechanical tensegrity model of the cell oocyte. Intrinsic properties of tensegrity elements are size, geometry and cell maturation dependent [39]. It explains the large discrepancy between experimental and simulation results. Changes in microtubule properties have the largest influence on the structural stiffness of the cell. This is attributed to their larger cross-sectional area, their positioning, and their role as compression bearing elements in the model. Furthermore, by increasing the number of microtubules, intermediate filaments and microfilaments in the tensegrity model would improve the force-deformation response but at a lack of real-time interaction in force feedback in the virtual environment.

TABLE III

COMPUTATION TIME VERSUS FINITE ELEMENT MESH REFINEMENT USING THE NONLINEAR MODEL

\begin{tabular}{cccc}
\hline \hline Vertices & Tetrahedron & $\begin{array}{c}\text { pre-computation time } \\
\text { off-line }(\mathrm{ms})\end{array}$ & $\begin{array}{c}\text { simulation time } \\
\text { on-line }(\mathrm{ms})\end{array}$ \\
\hline 115 & 332 & 0.875 & 0.047 \\
123 & 379 & 0.99 & 0.047 \\
128 & 407 & 1.094 & 0.063 \\
165 & 546 & 1.718 & 0.078 \\
176 & 614 & 2.031 & 0.094 \\
192 & 672 & 2.359 & 0.109 \\
271 & 979 & 4.546 & 0.156 \\
282 & 1019 & 4.859 & 0.156 \\
346 & 1269 & 7.39 & 0.187 \\
516 & 2130 & 18.828 & 0.328 \\
731 & 3060 & 36.5 & 0.453 \\
1093 & 4819 & 88.734 & 0.703 \\
\hline
\end{tabular}

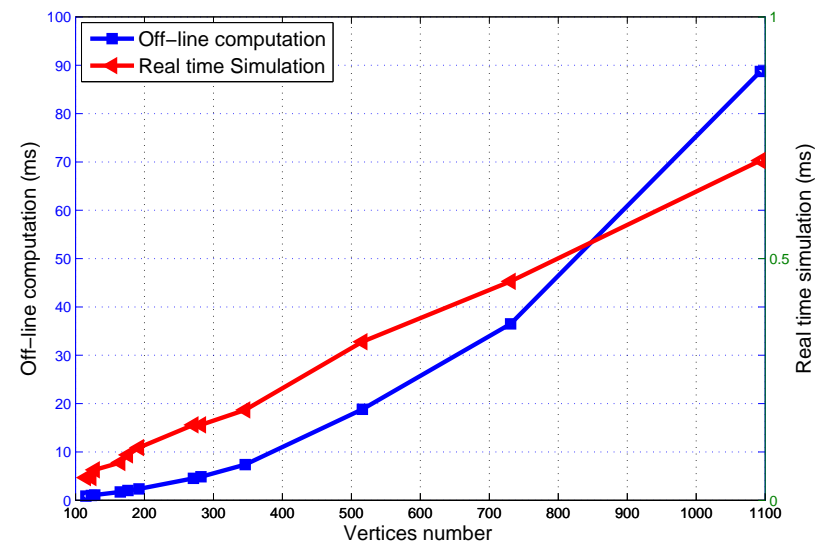

Fig. 14. Computation time versus vertices number: Pre-computation time and real-time simulation.

Finally, the coarseness of the mesh is strongly related to the accuracy of the end-result. As shown in Table.III, we can improve the real computational time by sacrificing accuracy. It is worth to notice that a computational accuracy of few $\mu m$ for insertion in nonlinear elastic and hyperelastic cells of diameter size of $60 \mu \mathrm{m}$, that is to say a maximum relative error less than $5 \%$ can be achieved at high haptic update rates on a standard PC. The discretization error caused by the coarseness of the mesh, relaxation errors caused by using an iterative algorithm and rounding errors taken together are below $1 \mu \mathrm{m}$. Preliminary results from this study suggest that the system is acceptable to a varied population of biologists but throughout its development, we will continue to evaluate the system for its efficacy in the resident training program.

\section{CONCLUSION}

We have developed a computer-based training system to simulate real-time cell micro-injection procedures in a virtual 
environment for training biologist residents. The simulator provides the user with visual and haptic feedback. The simulation procedure involves a real-time rendering through the haptic interactions with a physically-based model. We first investigated the challenging issues in the real-time modeling of the biomechanical properties of the cell micro-injection through finite element models. Compared to experimental data performed on oocyte cells, we can see clearly that the proposed physically-based FEM model is able to simulate the cell deformation through real-time simulation constraints. Currently, we are working on integrating others effects such as friction, viscosity, and adhesion forces. The simulator is prepared to doing an experimental training evaluation from trained and non-trained candidates. All modalities will be merged in an ergonomic and intelligent biological simulator to support learning micro-injection and training tasks.

\section{REFERENCES}

[1] T. Ebner, C. Yaman, M. Moser, M. Sommergruber, K. Jesacher, G. Tews, "A prospective study on oocyte survival rate after ICSI influence of injection technique and morphological features", J Assist Reprod Genet 18, pp. 601-606, 2001.

[2] S. Yu, B.J. Nelson, "Microrobotic Cell Injection”, IEEE International Conference on Robotics and Automation ,Seoul, Korea, , pp. 620-625, 2001.

[3] Y.H. Anis, M.R. Holl, D. R. Meldrum, Automated selection and placement of single cells using vision-based feedback control, IEEE Transactions on Automation Science and Engineering, Vol.7, No.3, pp.598-606, 2010.

[4] W.H. Wang, X.Y. Liu, Y. Sun, High-Throughput automated injection of individual biological cells,IEEE Transactions on Automation Science and Engineering, Vol.6, No.2, pp.598-606, 2009.

[5] A. Pillarisetti, M. Pekarev, A. D. Brooks, J. P. Desai, 'Evaluating the Effect of Force Feedback in Cell Injection", IEEE Transactions on Automation Science and Engineering, 2007, 4(3): pp. 322-331.

[6] H. Ladjal, J.L. Hanus and A.Ferreira, "Interactive Cell Injection Simulation Based on 3D bio-mechanical Tensegrity Model", IEEE International Conference on Robots and Intelligent Systems (IROS'08), September 22-26, 2008, Nice, France, Page 2296-2302.

[7] U. Meier, O. Lopez, C. Monserrat, M.C. Juan, M. Alcaniz, "Real-time deformable models for surgery simulation: a survey", Comput Methods Programs Biomed, 77(3), pp. 183-197, 2005.

[8] J. Kim, C. Choi, S. De, M.A. Srinivasan, "Virtual surgery simulation for medical training using multi-resolution organ models", Int J Med Robotics Comput Assist Surg, 3, pp. 149-158, 2007

[9] J. Kim, H. Ladjal, D. Folio, A. Ferreira and J.K. Kim, Evaluation of Telerobotic Shared Control Strategy for Efficient Single Cell Manipulation. IEEE Transactions on Automation Science and Engineering (T-ASE ), 9(2), pp. 402-406, ISSN 1545-5955. 2012.

[10] N. Bolhassani, R. Patel, M. Moallem , "Needle insertion into soft tissue : A survey", Med Eng Phys, 29 (4), pp. 413-431, 2007.

[11] M. Ammi, H. Ladjal, A. Ferreira, "Evaluation of 3D Pseudo-Haptic Rendering using Vision for Cell Micromanipulation", IEEE Intelligent Robots and Systems, Beijing, China, 2006, 2115 - 2120

[12] M. Asgari, A. Ghanbari, S. Nahavandi "3D Particle-based cell modelling for haptic microrobotic cell injection", 15th International Conference on Mechatronics Technology Melbourne, Australia, pp.1-6, 2011.

[13] V. Lulevich, T. Zink, H-Y. Chen, F-T. Liu, G-y. Liu, "Cell Mechanics using Atomic Force Microscopy-based Single-Cell Compression", Langmuir, vol. 22, no. 19, pp. 8151 -8155, 2006.

[14] H. Ladjal, J.L. Hanus, A. Pillarisetti, C. Keffer, A.Ferreira and J.P. Desai, "Reality-based real-time Cell Indentation Simulator", IEEE/ASME Transactions on Mechatronics, Vol. 17, Issue: 2, pp. 239 - 2502012.

[15] C.T. Lim, E.H. Zhou, S.T. Quek, Mechanical models for living cells - A review, Journal of Biomechanics, 39, 2, 195-216, 2006.
[16] T. Omori, T. Ishikawa, D. Barths-Biesel, A.-V. Salsac, J. Walter, Y Imai, and T. Yamaguchi, Comparison between spring network models and continuum constitutive laws: application to the large deformation of a capsule in shear flow, Phys. Rev. E, 83, 041918, 2011.

[17] Y. Tan, D. Sun, W. Huang, S-H. Cheng, Mechanical modeling of biological cells in microinjection, IEEE Transactions on Nanobioscience, vol.7, No.4, pp.257-266, 2008.

[18] Y. Tan, D. Sun, W. Huang, S-H. Cheng, Characterizing Mechanical Properties of Biological Cells by Microinjection, IEEE Transactions on Nanobioscience, vol. 9, NO. 3, pp.71-180, 2010.

[19] K. Kruse, J. F. Joanny, F. Jlicher, J. Prost, and K. Sekimoto, Generic theory of active polar gels : a paradigm for cytoskeletal dynamics, Eur Phys J E Soft Matter, 16(1), pp.5-16, 2005.

[20] F. Julicher, K. Kruse, J. Prostc b, and J.-F. Joanny. Active behavior of the cytoskeleton. Physics Reports, vol. 449, Issues 1(3) pp.3-28, 2007.

[21] D.E. Ingber, Tensegrity: the architectural basis of cellular mechanotransduction, Annual Review of Physiology,59 (1), pp.575-599, 1997.

[22] Y. Ujihara, M. Nakamura, H. Miyazaki, S. Wada, Proposed spring network cell model based on a minimum energy concept, Annals of Biomedical Engineering 38, pp.1530-1538, 2010.

[23] Y. Zeng, A.K. Yip, S.K. Teo, K.H.Chiam, A three-dimensional random network model of the cytoskeleton and its role in mechanotransduction and nucleus deformation, Biomechanics and Modeling in Mechanobiology, 11, pp.49-59, 2012.

[24] K. Wang, D. Sun, Influence of semiflexible structural features of actin cytoskeleton on cell stiffness based on actin microstructural modeling, Journal of Biomechanics, 45, pp.1900-1908, 2012.

[25] Y. Ruiguo, X. Ning, K.M.F.Carmen, S.S. Kristina, W.C.Lai. King, S. Bo, A.S. Animesh, Cellular tensegrity modeling with Atomic Force Microscopy (AFM) experimentation, IEEE Nanotechnology Materials and Devices Conference, pp.65-70, 2010.

[26] M. Mehrbod, M.R.K. Mofrad, On the significance of microtubule flexural behavior in cytoskeletal mechanics. PLoS One 6:e25627, 2011

[27] B. Maurin, P. Canadas, H. Baudriller,P. Montcourrier, N. Bettache, "Mechanical model of cytoskeleton structuration during cell adhesion and spreading", Journal of Biomechanics, vol.41, pp. 2036-2041, 2008.

[28] Y.S. Hee, L. ChanMo, M.R.K. Mofrad, Viscoelastic characterization of the retracting cytoskeleton using subcellular detachment, Applied Physics Letters, 98, 1337012011

[29] L. Lan, J.O. Sara, N. Hai, F.C.P. Yin, Mechanical properties of actin stress fibers in living cells, Biophysical Journal, Volume 95, Issue 12, pp. 6060-6071, 2008

[30] Y. Sun, K. Wan, K.P. Roberts, J.C. Bischof, B.J. Nelson,'Mechanical property characterization of mouse zona pellucida", IEEE Transactions On NanoBioscience, Vol. 2, No. 4, 2003.

[31] Y. Sun, B. J. Nelson, D. P. Potasek, and E. Enikov, A bulk microfabricated multi-axis capacitive cellular force sensor using transverse comb drives, J. Micromech. Microeng., vol. 12, no. 6, pp. 832-840, 2002.

[32] M.R.K. Mofrad and R.D. Kamm , Cytoskeletal Mechanics: Models and Measurements in Cell Mechanics (Cambridge Texts in Biomedical Engineering) Cambridge University Press, 978-0-521-84637, 2006.

[33] S.J. Peter, M.R.K. Mofrad , Computational Modeling of Axonal Microtubule Bundles under Tension. Biophysical Journal, Volume 102, Issue 4, 749-757, 22 February 2012

[34] T. Moller, B. Trumbore, "Fast, Minimum Storage Ray-Triangle Intersection”, Journal of graphics tools, vol. 2, no. 1, pp.21-28, 1997.

[35] J.G. McGarry, P.J. Prendergast, A three-dimensional finite element model of an adherent eukaryotic cell, European Cells and Materials, Vol. 7, pp.27-34, 2004.

[36] O.I. Wagner, S. Rammensee, N. Korde, Q. Wen, J.F. Leterrier, P.A Janmey, Softness, strength and self-repair in intermediate filament networks, Experimental Cell Research, 313(10), pp. 2228-2235, 2007.

[37] X. Liu, Y. Sun, W. Wang, B.M. Lansdorp, "Vision-based cellular force measurement using an elastic microfabricated device", Journal of Micromechanics and Microengineering, Vol.17, 2007, pp.1281-1288.

[38] X. Liu, R. Fernandes, A. Jurisicova, R.F. Casper, Y. Sun , In situ mechanical characterization of mouse oocytes using a cell holding device, Lab on a Chip, 10, pp. 2154-2161, 2010.

[39] J.S. Milner, M.W. Grol , K.L. Beaucage 3, S.J. Dixon, D.W. Holdsworth, Finite-Element Modeling of Viscoelastic Cells During High-Frequency Cyclic Strain, J. Funct. Biomater, 3, 209-224; doi:10.3390/jfb3010209, 2012. 


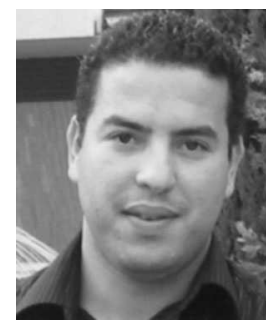

Hamid Ladjal received the M.S. degree in virtual reality and complex systems from Evry-Val d'Essone University, France, and the Engineer degree in electronics and computing from USTHB, and the Ph.D. degree in robotics and computer science from the University of Orlans, France, in 2010. He is currently an Associate Professor of computer science and biomechanical modeling at the Lyon Claude Bernard university, the image processing and information system (LIRIS CNRS UMR 5205), and the Institute of Nuclear physics of Lyon (IPNL CNRS UMR 5822), France. In 2009, he was a visiting researcher in the Robotics, Automation, and Medical Systems Laboratory, Maryland, USA. His research interests include mutliphysical, multiscale modeling and finite-element analysis, organ motion, reality-based soft-tissue modeling for real-time interaction, medical imaging and image processing, haptic interaction, cell characterization, and cell biomechanics, micro/nano manipulation.

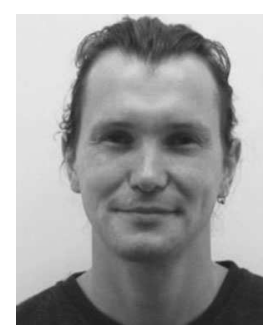

responses.
Jean-Luc Hanus received the M.S. degree from the National Aerospace and Mechanical Enginneering School, France, in 1993, and the Ph.D. degree in mechanical engineering from the University of Poitiers, Poitiers, France, in 1999. He is currently an Assistant Professor of mechanical engineering at the Ecole Nationale Suprieure d'Ingnieurs de Bourges, Bourges, France. His research interests include the area of nonlinear mechanical models and efficient numerical methods in dynamic analysis of biological cell and tissue

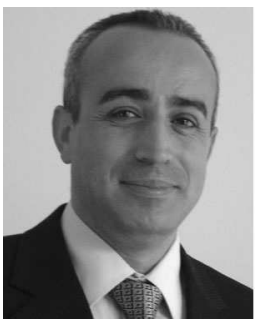

Antoine Ferreira (M'04) received the M.S. and $\mathrm{Ph} . \mathrm{D}$. degrees in electrical and electronics engineering from the University of Franche-Comt, Besancon, France, in 1993 and 1996, respectively. In 1997, he was a Visiting Researcher in the ElectroTechnical Laboratory, Tsukuba, Japan. He is currently a Professor of robotics engineering at the Laboratoire PRISME, Ecole Nationale Suprieure d'Ingnieurs de Bourges, Bourges, France. He is an author of three books on micro- and nanorobotics and more than 150 journal and conference papers and book contributions. His research interests include the design, modeling, and control of micro and nanorobotic systems using active materials, micro- and nanomanipulation systems, biological nanosystems, and bionanorobotics. Dr. Ferreira was the Guest Editor for different special issues of the IEEE/ASME Transactions on Mechatronics in 2009, International Journal of Robotics Research in 2009, and the IEEE Nanotechnology Magazine in 2008, IEEE Transactions on Robotics in 2013. He is actually associate editor in Reviews in Advanced Sciences and Engineering. 